\title{
MACEDONIO FERNANDEZ Y OMAR VIÑOLE: DOS CARAS DEL VANGUARDISMO EN ARGENTINA
}

Sabemos algo de Macedonio Fernández. Pero este otro: ¿un tal Omar Viñole? Cómo se justifica una propuesta temática que insinúa un parangón, aunque sea de signo contrario, ya que, como dice Bachtin, la literatura se asegura un espacio en la vida social por la conformación discursiva de las ideologías ${ }^{1}$, y en este caso, evidentemente, el tal Viñole parece no haberse asegurado nada, ni siquiera una placa conmemorativa dentro del espacio ideológico, como diría Zima, de la antiideología ${ }^{2}$. Removiendo las Historias de la literatura argentina, sean los seis tomos de la editada por Arrieta ${ }^{3}$, sean los 59 fascículos del Centro Editor ${ }^{4}$, o la Enciclopedia de la literatura argentina dirigida por Pedro Orgambide y Roberto Yahni ${ }^{5}$, el sujeto indicado no figura en ninguna de estas publicaciones que cubren la casi totalidad de las ideologías vigentes. Sólo en una publicación de la editorial "Todo es historia", que lleva el título Los excéntricos aparece, a la par de un artículo sobre un "diputado bromosódico" y "un mago de la lluvia", uno sobre "El hombre de la vaca", quien es, efectivamente, nuestro Omar Viñole 6 .

La omisión de éste, siendo autor de numerosos libros ${ }^{7}$, podría invitar a una reivindicación explícita incorporándolo en el ámbito de los marginados, hasta ahora - pienso en Luis Pascarella, Ismael Moreno y Juan Filloy - o de aquellos que con atraso fueron rehabilitados en su singularidad - desde José Hernández hasta Juan

1 Cf. Carlos Altamirano, Beatriz Sarlo, Literatura/Sociedad. Buenos Aires 1983, p. 33.

2 Cf. Peter V. Zima, Ideologie und Theorie. Tübingen 1989.

3 Historia de la literatura argentina. Buenos Aires 1958-60.

4 Capttulo. La historia de la literatura argentina. Buenos Aires 1967-68.

5 Buenos Aires 1970.

6 Ernesto Goldar, "El hombre de la vaca", en: Los excéntricos. Buenos Aires 1977, pp. 53-78.

7 Cf. Anotacion núm. 28. 
L. Ortiz - y abrir el proceso contra un sistema de valores que pocas veces fue cuestionado con el debido rigor. Para una empresa reivindicatoria de esta amplitud los parámetros serían otros que los que impone la temática general del vanguardismo, que invita, sin embargo, a arriesgar la hipótesis de la incompatibilidad estructural de vanguardismo y lo que Peter Bürger llama "Institution Kunst" ${ }^{\prime \prime}$. Esto es algo diferente de la constatación del fracaso de las vanguardias históricas al ser absorbidas en un momento dado por la "Institution Kunst" para, a su vez, erigirse en modelos normativos. Si sugerimos una incompatibilidad estructural, estamos relativizando todas estas manifestaciones que se integran en el sistema de valores estéticos como estructuralmente destinadas para tal integración. Se trata, por supuesto, de una abstracción que sólo podría verificarse tendente y gradualmente. En los casos de Macedonio Fernández y Omar Viñole vislumbro ejemplos que podrían convalidar la hipótesis, en el sentido de contener el discurso de este último todavía más negativas al sistema de valores establecidos que el de Fernández.

Cautelosamente hablo del vanguardismo en Argentina, estableciendo un marco geográfico social cuya extensión temporal podría fijarse entre 1915, cuando aparece - mejor dicho, desaparece - El cencerro de cristal de Ricardo Güiraldes, y 1954, año de publicarse En la masmédula de Oliverio Girondo, admitiendo que el lustro entre 1922 y 1927, cuando se nuclea un nutrido grupo de escritores alrededor de las revistas Proa y Martín Fierro, es el de mayor densidad y virulencia. Si la vanguardia histórica, en el modelo que propone Bürger, significaba una ruptura radical con la "Institution Kunst" que había encontrado su conjunción mas nítida y acendrada en el esteticismo como sellado del divorcio de la práctica artística y práctica vital, hay que suponer que en Argentina esta ruptura que tendría que implantar no solamente un nuevo estilo sino un cambio profundo de la concepción del arte, se da con bastante timidez.

Si analizamos el manifiesto más importante del ultraísmo ${ }^{9}$ argentino, redactado por Oliverio Girondo y publicado en la revista más descollante de esta promoción, Martín Fierro ${ }^{10}$, podemos admitir que

8 Cf. Peter Bürger, Theorie der Avantgarde. Frankfurt am Main ${ }^{2} 1980$.

9 Emplearé en este trabajo sin distinción los términos "ultraístas" y "martinfierristas".

$10 \mathrm{I}, \mathrm{n}^{2} 4$,. Mayo de 1924. 
éste, por "su sintaxis y su orden fonológico, por su estratégica ruptura del orden lógico y su apariencia no convencional [...] ofrece una forma nueva de discurso para la tradición literaria argentina"11. Pero si comparamos las propuestas concretas del manifiesto, con las posiciones teóricas más avanzadas del modernismo, que podrían ser las "Dilucidaciones" con que prologa Rubén Darío su libro de poemas El canto errante (1907), comprobamos que los detalles concordantes, además de la reverencia que se le brinda a Darío en el manifiesto del Martín Fierro ${ }^{12}$, exceden, cuantitativamente por lo menos, los desacuerdos eventuales que no pasarían de matices estilísticos. La proclamada "nueva sensibilidad" enjuicia a los conocidos fantoches de un ignorante público mayoritario y a los académicos y escritores esclerotizados, pero no se cuestiona fundamentalmente la función de la obra artística. La finalidad de ella a nivel social, entre los polos de la autonomía o de la integración en la práctica vital, y el modo de la producción así como el de la recepción, no figuran conceptualmente en el manifiesto. Por lo demás es presumible que el propio Rubén Darío hubiera admitido sin reticencia las aseveraciones de los ultraístas argentinos con respecto a la originalidad creativa:

MARTIN FIERRO artista, se refriega los ojos a cada instante para arrancar las telarañas que tejen de continuo: el hábito y la costumbre. iEntregar a cada nuevo amor una nueva virginidad, y que los excesos de cada día sean distintos a los excesos de ayer y de mañana! iEsta es para él la verdadera santidad del creador! ... iHay pocos santos! ${ }^{13}$

David Viñas quien enfoca el movimiento martinfierrista desde el ángulo de su eficacia histórico social, denuncia una actitud que oscila entre innocuidad y acomodación:

11 Francine Masiello, Lenguaje e ideología. Las escuelas argentinas de vanguardia. Buenos Aires 1986, p. 71.

12 R. Darío fue el primero quien saludó al futurismo de Marinetti en un artículo publicado en La Nación de Buenos Aires. Cf. Nelson Osorio T., "Antecedentes de la Vanguardia literaria en Venezuela (1909-1925)". En: Hispamérica, 33 (1982), p. 5 .

13 Martín Fierro, I, n ${ }^{\circ}$, mayo de 1924. A su vez, hasta los martinfierristas más rebeldes, hubieran firmado el cierre de las "Dilucidaciones" que reza: "No hay escuelas; hay poetas. El verdadero artista comprende todas las maneras y halla belleza bajo todas las formas. Toda la gloria y toda la eternidad están en nuestra conciencia." (Rubén Darío, Poesías completas. Edición, introducción y notas de Alfonso Méndez Plancarte. Madrid ${ }^{10} 1967$, p. 700). 
Con los viejos, caducos e instalados, se practica un terrorismo suculento, sobreactuado o juguetonamente irónico de manera que se entienda - por exceso o por distancia - que la cosa no pasa de ahí y sólo se trata de ser reconocido por el otro en ese juego bonachón e impertinente. En el fonđo los martinfierristas siempre presintieron que su pasaje de lo juvenil sería un salto a la vejez $[\ldots]^{14}$

El enjuiciamiento aplastante de Viñas coincide curiosamente con la opinión de Jorge Luis Borges para quien el martinfierrismo se reduce a "inocentes novedades ruidosas" de algunos muchachos "tímidos" y"temerosos de una íntima pobreza"15 habiendo reconocido ya en 1937 - expiando los propios pecados $-{ }^{16}$ que el martinfierrismo "no era más que el desarrollo parcial de una de las vertientes abiertas por Lugones en el Lunario sentimental"17.

Me ahorro el trabajo de citar otros juicios negativos sobre el martinfierrismo que relativizan su incidencia en la vida cultural de Argentina a las travesuras de unos hijos de buena familia o al folklore del Buenos Aires de los años locos y de bonanza, durante la presidencia de Alvear. Estoy de acuerdo con Viñas al denunciar la falta de arrojo y pertinacia del movimiento en su totalidad. El martinfierrismo no pasa de ser un reflejo, inclusive algo pálido, de lo que se venía dando en Europa. Por otra parte hay que suponer en Argentina una mayor inamovilidad de las estructuras sociales y, por lo tanto, más obstáculos y resistencia de las fuerzas conservadoras que en los países europeos sacudidos por la primera guerra mundial. El famoso mingitorio firmado por Marcel Duchamp ${ }^{18}$ y presentado en una exposición de arte en 1917 hubiera sido inconcebible en la Argentina de entonces cuando El cencerro de cristal (1915) de Ricardo Güiraldes, un poemario que refleja cierta influencia de Apollinaire,

14 David Viñas, Literatura argentina y realidad politica. De Sarmiento a Cortázar. Buenos Aires 1971, p. 63.

15 Jorge Luis Borges, Obras completas. Buenos Aires 1974, p. 13.

16 Pienso en los ataques polémicos a varios de los escritores consagrados, incluidos en sus libros de ensayos, Inquisiciones (1925) y El tamaño de mi esperanza (1926) que nunca fueron reeditados y tampoco figuran en las Obras completas.

17 Adolfo Prieto, "Prólogo" a su Antología de Boedo y Florida, Córdoba 1964, p. 14.

18 Cf. P. Bürger, op.cit., p. 70. 
fue tan implacablemente censurado que el autor prefirió enterrar la edición en un pozo de su estancia ${ }^{19}$.

Admitiendo, pues, su especificidad social e histórica, consta que el movimiento martinfierrista no podía llegar a adquirir un perfil más radical, y que las tibiezas del "Manifiesto" se deben a la estrategia de unir a todos aquellos que por cualquier razón, y no solamente por el nuevo programa estético, se oponían a los valores y poderes establecidos. Hasta ahora siempre se ha visto dentro del grupo martinfierrista dos posiciones diferentes que emergen, cuando en 1927 se formó el Comité Irigoyenista de Intelectuales Jóvenes ${ }^{20}$ cuyo apoyo a la reelección de Yrigoyen llevó al cierre de la revista por los directores, Evar Méndez y Oliverio Girondo, que sostuvieron la separación de las esferas del arte y de la política. Pero es evidente que en el propio campo de la producción literaria se enfrentan dos posiciones que se diferencian por el grado de sus transgresiones con respecto a las normas vigentes. En primer lugar hay que mencionar a Oliverio Girondo quien, según Francine Masiello, "se destaca como el exponente principal de esta reforma estética" (luego habla de "revolución artística"), cuyos Veinte poemas para ser leídos en el tranvía (1922) "introdujeron en la Argentina la orientación ultraísta, forma tan a menudo adjudicada a Borges. Con esta obra Girondo postuló la necesidad de un cambio lírico que escandalizó al público lector" ${ }^{\prime 2}$. Puede dudarse que este libro realmente escandalizara al "público lector", y no, exclusivamente a los representantes de la cultura oficial. De todos modos, se trata de una manifestación quizá no revolucionaria, pero audaz y rebelde, y lo más escandaloso, al nivel de un ataque a la "Institution Kunst", es el famoso epígrafe de: "Ningún prejuicio más ridículo, que el prejuicio de lo SUBLIME." Con esto, sí, Girondo liquidó, o intentó liquidar, en un gesto de formidable arrogancia la piedra angular de la estética precedente. "Lo sublime".

19 Sin el menor intento de argumentar, el crítico más importante del momento - Julio Noé - y en la revista más importante - Nosotros (n 85, 1916) - lo declaró "francamente malo" agregando la advertencia: "Lástima que el señor Güiraldes quiera, a todo trance, ser original y suponga que aún hay burgueses a épater."

20 El cual integran los hermanos González Tuñón, Borges, Marechal, Pondal Ríos, López Merino y algunos más. Cf. Carlos Mastronardi, "El movimiento de 'Martin Fierro’", Captululo. La historia de la literatura argentina, № 39, Buenos Aires 1967 , p. 928.

21 Francine Masicllo, "Oliverio Girondo: El carnaval del lenguaje." En: Hispamérica, 16 (1977), p. 4. 
Lo que, etimológicamente, "subleva", lo que es de "gran belleza y tal que provoca una emoción noble" 22 , fue reducido a una cosa de mal gusto. Lo que Girondo combate es lo que conserva o reivindica Borges en Fervor de Buenos Aires (1923). Las oposiciones son, por lo menos, las siguientes: la visión caótica del mundo frente a la visión enternecida del barrio; indecorosidad de imágenes y palabras frente a una petulante observación de la decencia; un yo poético, hasta cierto punto deshumanizado, frente a un yo emocional y biográficamente comprometido. Finalmente hay que destacar, en el caso de los Veinte poemas, la desacralización del hecho literario al invitar a una lectura que no quiera ser distinta de la lectura de un diario.

Por otra parte, los Veinte poemas, en la edición de 1922 una verdadera joya para bibliófilos, se instala como tal rotundamente en el ámbito de la cultura tradicional, desde la repetida afirmación de la individualidad autorial como productor e ilustrador de los textos hasta el colofón, donde el autor vuelve a aparecer como firmante de los 150 ejemplares "puro hilo Lafuma, fuera del comercio", para no hablar del lugar de la impresión, Argenteuil, que distaba mucho de ser Avellaneda u otro suburbio de Buenos Aires. Además, ya una somera comparación con Trilce de César Vallejo, publicado en el mismo año de 1922 en Lima y en los ciertamente no muy prestigiosos "Talleres tipográficos de la Penitenciaría", evidencia las limitaciones de la ruptura de Girondo con los vigentes cánones estéticos, por lo menos respecto de la inteligibilidad discursiva y la representación mimética. El hecho de haber encontrado el libro de Girondo una acogida más atenta en Lima que en Buenos Aires $^{23}$, permite apreciar la dimensión real de la "impermeabilidad hipopotámica del 'honorable público" argentino, el primer objeto de las imprecaciones del manifiesto martinfierrista.

Fernando Alegría define la "antiliteratura" en los términos de

[...] una revuelta contra una mentira aceptada socialmente y venerada en vez de la realidad. Esta antiliteratura empieza por demoler las formas, borrar las fronteras de los géneros, dar al lenguaje su valor real y corresponder con sinceridad a la carga

22 Según el Diccionario de uso del español por María Moliner, Madrid 1984.

23 Lo que consta del número de las reseñas. En Lima aparecieron 5 reseñas firmadas por A. G., Pedro Barrantes Castro, Federico Bolaños, "Clovis" y F. B.; en Buenos Aires 3 reseñas firmadas por el español Enrique Diez Canedo, Vicente Martínez Cuitiño y el español Guillermo de Torre (en Proa, 12, 1925). 
de absurdo que es nuestra herencia. Lo blasfemo, así como lo irreverente, insultante y hasta lo obsceno, son modos de aclararle al hombre el espejo donde está su imagen. $^{24}$

Alegría circunscribe el fenómeno principalmente a la literatura surgida en los años 60, con algunos antecedentes en los años 30 . Su definición sirva aquí ni como categoría analítica, ni para trazar una línea evolutiva, sino para fijar una pauta evaluativa. Si la aplicamos al movimiento martinfierrista en su totalidad, con adyacentes, quizá, fuera de Oliverio Girondo, el "Peter Pan de la vanguardia", como lo titula Viñas ${ }^{25}$, sólo hay uno quien - reconocidamente - se destaca por su actitud constante de cuestinar los presupuestos de la literatura: Macedonio Fernández. Es cierto y no se debe olvidar que por más efímeras que resultaran las manifestaciones del grupo que se realizaron en el espacio de lo no-sublime - su irrespetuosidad por algunos de los valores establecidos, su actitud lúdica, sus intentos de alcanzar e interesar un amplio público por los debates acerca de la función del arte, etc. - éstas definieron tendencialmente un nuevo clima cultural al que Macedonio Fernández debe impulsos decisivos. El mismo - con cierta ironía - lo reconoció:

Por culpa de la juventud artística de Buenos Aires que conocí hace cuatro años [en 1925] estoy abismado en un problema de estética. Me desvalijaron por aquel entonces con tanta prolijidad e inmenso provecho de mi estética pasatista que hasta la fecha no he podido recuperar una ignorancia igual. ${ }^{26}$

Dije que hay uno, reconocidamente, quien realizó el proyecto vanguardista al nivel de una ruptura con los presupuestos de la literatura comprendida en su funcionamiento institucional, cuya validez, muy poco después de las "travesuras" juveniles, fue con-

24 Fernando Alegría, "Antiliteratura", En: César Fernández Moreno (ed.), América latina en su literatura. México, París 1972, p. 243.

25 Op.cit., p. 65.

26 Cit. por el "Prólogo" de Ramón Gómez de la Serna. En: Macedonio Fernández, Papeles de recienvenido. Buenos Aires 1944, p. 17. Waltraut Flammersfeld, Macedonio Fernández (1874-1952). Reflexion und Negation als Bestimmungen der Modernität. Frankfurt, Bern 1976, afirma: "Es darf nicht verschwiegen werden, daß trotz aller Unzulänglichkeiten der Avantgarde die Begegnung mit ihr für MF zum Auslöser seiner ästhetischen Reflexion wurde [...] In der Tat ist die Ausformung der avantgardistischen Kunsttheorie MFs nach den vorhandenen Texten eindeutig ab 1925 zu datieren." (p. 54). 
solidada hasta el punto de ver en una novela como Don Segundo Sombra - consagrada por Leopoldo Lugones - la obra cúspide del ultraísmo y en Macedonio Fernández un mito, una leyenda viva, un metafísico, un guitarrero, pero al fin un espécimen "a quien", según su admirador Borges, "no le interesó la literatura" ${ }^{27}$ y por lo tanto no perteneciente a ella. Hay, o presumo que hay otro, no reconocido: Omar Viñole, médico, veterinario, candidato a gobernador de la provincia de Córdoba, luchador del "catch", prontuariado por varias incidencias con la policía, la mayoría de ellas perpetradas mediante una vaca, pintor y autor prolífico ${ }^{28}$.

Si el discurso de Macedonio Fernández - y uso el concepto de discurso en un sentido que englobe no solamente la totalidad de sus textos sino también las actitudes que conformaron su personalidad y su mito - se caracteriza en una de sus vertientes principales por un encadenamiento de negaciones, puede afirmarse lo mismo del discurso de Viñole. El sistema filosófico de Fernández, que tiene su principio totalizador en lo que él llama "almismo ayoico"29, se construye negando las categorías gnoseológicas y ontológicas. Ya en No toda es vigilia la de los ojos abiertos (1928) se afirma:

El Yo, Materia, Tiempo y Espacio, son los faltantes en el Mundo: el genio gramatical puede sustantivarlos así con un vocablo que precisamente los niega como substancias y como fenómeno. ${ }^{30}$

Paralelamente abarca el "belarte conciencial de la palabra" como designa Fernández su concepción de la literatura, una serie de negaciones que no solamente se refieren a las convenciones estéticas y miméticas, sino - en la dimensión pragmática - a los roles convencionales de autor y lector y, finalmente, al cuestionamiento de la publicación de la obra literaria.

En el caso de Omar Viñole puede constarse una semejante negatividad y además en los mismos campos: filosofía y literatura, cuya institucionalización oficial en forma del academismo sería en

27 Cf. Flammersfeld, op.cit., p. 57.

28 Goldar indica "casi 60 monografías de carácter científico" y "43 libros polémicos", op.cit., p. 66. Ver Apéndice.

29 Cf. Flammersfeld, op.cit., p. 45.

30 Macedonio Fernández, No toda es vigilia la de los ojos abiertos. Buenos Aires 1928, p. 48. 
ambos casos el objeto predilecto de sus ataques irónicos, parodísticos o, sobre todo en el caso de Viñole, injuriosos:

La resignación atrofia. Ved a esa Academia de Letras argentinas. Los que están allí, son los resignados. Exploradores de regreso. Se juntan de tarde en tarde para disfrutar de una compañía que atenúa la tristeza de estar solo en el fracaso. (Cien cabezas, p. 11)

El discurso de Macedonio Fernández permite inferir una opinión idéntica, aunque por cuestiones, digamos de decoro, no la hubiera publicado $^{31}$.

No puedo entrar aqui en los detalles de los respectivos sistemas filosóficos, lúcidamente - como sistema - reconstruido y analizado por Waltraud Flammersfeld en el caso de Macedonio Fernándezy en absoluto elaborado, ni siquiera registrado en el caso de Omar Viñole. Uno de los libros de éste se titula Mi disconformismo filosófico ${ }^{32}$. Ahí, en su manera poco meticulosa el autor jaranea: "La felicidad no existe" (pp. 24-25) "La ciencia no existe" (pp. 29-32), "El hombre no existe" (pp. 31-33), etc. Si perseguimos este "disconformismo filosófico" hasta llegar a una concepción totalizadora, podemos inferir que Viñole, interrogado por la relación entre el "ser" y la percepción del "ser", se remitiría a una posición netamente materialista. El hombre no es sino

[...] un inquilino transitorio que se enferma en gonorrea e incuba otras enfermedades además de la lues, que está condenado a muerte, que tiene un 60 por ciento de agua y el remanente pertenece a extractos secos, albúminas, o proteínas, sales de cloro, de hierro, el fósforo, la cal, el yodo, el manganeso, el cobre y el aluminio.

Este diagnóstico está firmado por "Jesús de Nazareth (alias) Jesucristo" ${ }^{\prime 33}$. Es evidente que esta concepción de la condición humana es una provocación satírica que implica en primer lugar la negación de la especulación metafísica. Es probable que la filosofía de Viñole no tenga mayores alcances, buscando el origen ontológico

31 El antiacademismo de Macedonio Fernández se articula en una gama muy amplia de ironizaciones y parodias, por ejemplo en varias de sus "Teorías", de la "salud", del "Estado", etc. Cf. Flammersfeld, op.cit., p. 111.

32 Cf. Apéndice.

33 Omar Viñole, Jesús en una casa de departamentos. p. 22. El ejemplar del que dispongo tiene una dedicatoria ológrafa: "A Oliverio Girondo, nos veremos en el infierno. Omar". 
de la cognición, lo cual sería una consequencia más lógica - aunque, por supuesto, poco satisfactoria - que una construcción como el "almismo ayoico" de Macedonio Fernández, al fin producto de la especulación metafísica aunque fuera esta misma el objeto de su crítica.

Si parangonamos, ahora, las actitudes de los dos con respecto a la literatura en las dimensiones de la teorización, las propias concretizaciones y la posición frente a la literatura como institución, observamos varias coincidencias, con las obvias diferencias temperamentales que los enfrentan irreconciliablemente. Macedonio Fernández, hasta en los accesos más demoledores, presenta sus insolencias con moderación, irritando, no obstante hasta a amigos como Borges $^{34}$. En Papeles de Recienvenido, pero no en la primera edición de 1930, tampoco en la segunda de 1944, sino sólo en la póstuma de 1966, arremete contra lo consagrado en los siguientes términos:

Todos los trabajadores artistas han mostrado antojos raros en sus horas laboriosas: Victor Hugo, que escribía un libro por año, no se sentía fuerte para comenzarlo hasta que no había concluido de vivir todo ese año sin pensar en nada [...] Balzac no empezaba a escribir sin tener cerca de sí la ausencia en viaje a Europa de su suegra (ya se entiende que el viaje a Europa de una francesa es venir a la Pampa); Colón no descubría continentes, o lo hacía enteramente de mal humor, si no se los ponían por delante impidiéndole seguir la redondez hasta la Asia [...] a Gautier el vacío en la cabeza era la sensación sin la cual no podía llenar la primer página [...] y el ocioso Byron al comenzar a trabajar no hacía nada: pasaba tantos años sin reflexionar en cosa alguna que cuando quería retratarse no acertaba con la postura de pensar. ${ }^{35}$

Viñole se esmera menos, según uno de sus innumerables lemas: "El talento como los granos en el culo, impide sentarse donde uno desea." (La camiseta, p. 41.) Un pequeño florilegio viñoleano, cuyos enunciadores pueden identificarse con el autor, abarca las siguientes injurias:

"(A)quel senil impotente que se llamó Anatole France" ( $L a$ camiseta, p. 64), "Kant mismo no fué nada mas que un liliputiense reformador de Hume" (El hombre, p. 78), "el sublime pederasta Oscar

34 Cf. Flammersfeld, op.cit. p. 59.

35 M. F., Papeles de Recienvenido. Buenos Aires 21967, p. 77s. 
Wilde decía que la gratitud es denigrante" (Cien cabezas, p. 11), "el tilingo Gómez de la Serna" (El hombre, p. 101) ${ }^{36}$.

Sobre todo arremete Viñole contra los escritores consagrados de Argentina, "los chocolateros de la literatura" (Jesús, p. 114), Capdevila, Lugones ("escritor a pesebre" o "globetroter ideológico" (Jesús, p. 3, 74)), Manuel Gálvez o Enrique Larreta - "Tambien es fiambre La gloria de Don Ramiro que fué escrita hace trescientos años por un cura español" - (Cien cabezas, p. 11). En un capítulo de El hombre que se depiló la ingle, cuyo protagonista es Oscar Rubén Darío Vagagá, se comparan vacas y escritores:

Zootécnicamente considerado, el escritor puede dar de catorce a treinta litros [de leche]. Por ejemplo, Lugones (el papá) es de treinta litros. César Carrizo, de unos once litros. Barón Biza de seis a siete litros [...] Bromatológicamente, no podríamos llamar leche ni aun a las producciones de Constancio Vigil, porque casi todo su contenido está en el padre nuestro. (pp. 93 ss.)

Al padre del protagonista, el narrador lo trata con un secreto respeto:

[...] un señor Rubén Darío, que tenía algo en la cabeza, además de la caspa, y que se pasó la vida borracho, como única forma de mantener la ilusión de ser comprendido en la verdad, y que además de esto escribía versos, algunas veces con fines urinarios, otras para conseguir algo de dinero y 'mamarse' (vulgo curda). (p. 39)

Aunque Fernández y Viñole se distinguen fundamentalmente en su actitud de publicar - el primero lo hacía a instancias de sus amigos y con parquedad, el otro se da incansablemente, con sus palabras, "el ambigú de excitar la cólera, hasta que los cabrones caigan en colapso" (El Hombre, p. 37), publicando en la decada del 30 casi tres libros por año - ambos concuerdan en el escepticismo con respecto de la literatura, es decir de los presupuestos de la literatura institucionalizada, con cierta salvedad, tambien compartida por los dos, considerando a su propia poesía lírica como, en el caso de Fernández, "Aussage tiefer, begrifflich nicht faßbarere Wahrheiten" ${ }^{37}$, en el caso de Viñole, algo semejante, cuando confiesa en el epígrafe de Alambres de yeso, Poemas en Verso: "Libro, yo te excomulgo, porque delatas la

36 Los que se salvan de las diatribas de Viñole son Einstein (cf. Jesus, p. 106), Ramón J. Cárcano, Girondo, Filloy, Botana y algunos más (cf. Cien cabezas).

37 Flammersfeld, op.cit., p. 32. 
tristeza del otro Omar Viñole, OMAR VIÑOLE." Para éste, "ningún hombre serio de verdad escribió nunca! Al hombre serio le basta y le sobra con vivir el drama en cuatro actos de su seriedad." (El hombre, p. 56).Preguntado por su interlocutor ficticio, ... "ipor qué escribes?", el portavoz del autor contesta: "iCon el único fin de desprestigiar las letras!" (El hombre, p. 73))

La crítica de la literatura por Fernández y Viñole se centra parecidamente en el mimetismo, retomando los dos, sin reconocerlo explícitamente, el principio del creacionismo de Vicente Huidobro (más radical que el conjunto de las propuestas programáticas del martinfierrismo). En su lenguaje poco remilgado profesa Viñole (por boca de Vagagá):

$\mathrm{El}$ arte es un revulsivo que le pasan a las colectividades burguesas, para que les pique el ojete y salgan de casa [...] El arte está en la naturaleza. Copiarla significa embetunarla, orinarla, cagarla, estrangularla y perturbarla. (El hombre, p. 47)

Aunque en el caso de Macedonio Fernández la meta sea distinta, lo que aún debe discernirse, sus referencias críticas al "realismo" o a la "copia" son tan repetidas ${ }^{38}$ que puedan considerarse uno de los fundamentos de su estética que se cifra en el concepto del "belarte conciencial". En uno de sus ataques más explícitos, que quizás entraña la noción de "Widerspiegelung", declara:

Todo el realismo en arte parece nacido de la casualidad de que en el mundo hay materias espejeantes; entonces a los dependientes de tiendas se les ocurrió la Literatura, es decir confeccionar copias. Y lo que se llama Arte parece la obra de un vendedor de espejos llegado a la obsesión, que se introduce en las casas presionando a todos para que pongan su misión en espejos, no en cosas. En cuántos momentos de nuestra vida hay escenas, tramas, caracteres; la obra de arte-espejo se dice realista e intercepta nuestra mirada a la realidad interponiendo una copia. ${ }^{39}$

Las negativas de Fernández se refieren globalmente a uno de los principios fundamentales de la literatura, la verosimilitud ${ }^{40}$. Además especifican, al mencionar a "escenas", "tramas" y "caracteres", como

38 Cf. Noé Jitrik, "La 'Novela futura' de Macedonio Fernández. En: N.J., El fuego de la especie. Buenos Aires, Mexico, Madrid 1971, p. 161.

39 Macedonio Fernández, Museo de la novela de la Eterna. Buenos Aires 1967, p. 109.

40 Cf. N. Jitrik, op.cit., p. 162.

41 Se publicó por primera vez en Buenos Aires, 1974, como tomo V de las Obras completas de Macedonio Fernández. 
objetos de la realidad e impropios para una nueva y "buena" literatura, los elementos imprescindibles para cualquier texto narrativo "convencional" o "malo", como lo sería Adriana Buenos Aires, la "última novela mala" ${ }^{41}$. La concretización de un texto no-realista vendría a ser "la novela de la Eterna", cuyos materiales constituyen el texto que fue publicado con el título de Museo de la novela de la Eterna ${ }^{42}$. La idea primitiva, según el editor Adolfo de Obieta, "fue narrar toda la novela en prólogos [...] Después, algunos prólogos se transmutaron más o menos libremente en 'capítulos' y la novela propiamente dicha comenzó a enterizarse" ${ }^{\prime 43}$. No intentaré aquí un acercamiento a la teoría estética o la teoría del "personaje" de Macedonio Fernández, concretizada en ese texto como una constante búsqueda que, dadas las dimensiones de ella, remite, ante todo, al proceso y no al resultado. En cuanto a las concomitancias de las concepciones literarias de Fernándezy Viñole, pueden observarse en la práctica literaria de este último sorprendentes analogías o inclusive idénticos procedimientos al nivel formal.

El más evidente es lo que Goldar llama "la desmesura de los prólogos" ${ }^{\prime \prime 4}$, que en el caso, por él mencionado, de Como vienen al mundo las palabras ocupan más de la cuarta parte del libro. En El hombre que se depiló la ingle se adelantan unas 33 páginas, digamos preparatorias, a un texto de 67 páginas, en que aparece como personaje enunciado, narrador en primera persona e interlocutor de otro narrador en primera persona, el sujeto que se dedica profesionalmente a la actividad indicada en el título. Las páginas introductorias consisten en: una dedicatoria, una explicación del "cometido", una lista de publicaciones, un "Prólogo", una "Carta de Omar Viñole a S. S. el papa", una instrucción "El arte de matar ratas", varias otras advertencias o confidencias al lector, una receta para "preparar el engrudo", un reglamento para una "pileta literaria", y otros proemios, la mayoría de todo este material firmada por "Omar Viñole" o "El autor", a veces con un agregado, como "Sin sinonimia", "Se suplica denigración" o "iDisculpen! iEsta meditación se me cayó! iFué sin querer!" (p. 36)

42 Buenos Aires 1967.

43 M. F., Museo, p. 5.

44 Goldar, op.cit., p. 57. 
Aunque en El hombre que se depiló la ingle o en Jesús en una casa de departamentos ${ }^{45}$ figuran personajes protagonistas - y no, como en La camiseta del jefe de policía sujetos inanimados que exponen las reflexiones del autor (implícito) - e inclusive un escenario cuyo referente permite cierta concretización, es evidente que se trata de procedimientos empleados como trampas para eventuales lectores ingenuos, tan notoriamente que no sólo denuncian el ilusionismo literario como tal, sino - en un nivel más astuto u original - denuncian la ficcionalidad del lector ingenuo que pudiera caer en la trampa. Si el firmante "Jesús de Nazareth" se dirige "A los lectores que no creen que Jesús ha estado en Buenos Aires" o si en El hombre que se depiló la ingle en un esguince imprevisto se plantea: "¿Dónde aprendió a ser hijo de puta Vagagá? ¿Lo preguntáis, lectoras?" (p. 40) - queda establecida la diferencia fundamental entre la instancia del lector como objeto del discurso y el destinatario real. Al cual a lo mejor no se le hubiera ocurrido la última pregunta.

A modo de epílogo vuelvo a la hipótesis más arriba arriesgada, de la incompatibilidad estructural entre "Institution Kunst" y vanguardismo. El intento de suspender la distancia entre el arte y la práctica vital en el cual cifra Bürger la empresa principal del vanguardismo histórico ${ }^{46}$, probablemente en el momento de perder su carácter de intento y concretizarse como práctica vital, es decir en el espacio público de una sociedad, se excluye del espacio artístico. Si Macedonio Fernández emprende, en 1927, irónicamente una campaña presidencial a su favor y proyecta, como cuenta César Fernández Moreno:

[...] crear un verdadero malestar general, para suscitar la necesaria venida de un gran caudillo que lo conjurara, o sea el propio Macedonio. Medidas concretas propuestas por él en ese sentido eran: repartir peines de doble filo, que lastimaran el cuero cabelludo [...] instalar salivaderas oscilantes [...] solapas desmontables [...] escaleras desparejas [etc. ${ }^{47}$

45 En Jesís en una casa de departamentos, los prólogos (48 páginas de un total de 190) están firmados alternativamente por "Omar Viñole" y "Jesús de Nazareth" que a veces agrega un "(álias) Jesucristo".

46 Bürger, op.cit., p. 68.

47 César Fernández Moreno, Introducción a Macedonio Fernández. Buenos Aires 1960 , p. 10 s. 
- nada de esto se realiza. Es decir, esta campaña, por irreal, mantenía su aura artística y podía integrar el mito de la genialidad de Macedonio Fernández. Viñole, por otra parte, que arremete contra casi todo - iglesia, literatura, municipalidad, el fachismo, los ingleses, la prensa oligárquica, etc. - y, por colmo, exhibe sus insolencias públicamente paseándose con una vaca real por las calles de Buenos Aires, retando públicamente el fachismo vernáculo, etc. fue una presencia demasiado corpórea, estridentemente física, así que - hasta ahora - se le ha negado el ingreso al empíreo de las letras ${ }^{48}$.

\section{APENDICE}

(Libros publicados por Omar Viñole entre aproximadamente 1930 y 1940, por orden cronológico.)

Escritos y Cuentos camperos. Prosa.

Psicología de los que van al cine.

Omar. (Poemas Tagorianos)

Cristóbal Colón de origen luético. Pieza teatral

Biología Sentimental. Pieza teatral.

Tuberculosis bovina. Est. técnico.

Mapa Pomológico de la R. Argentina. Est. técnico.

Las primeras experimentaciones de Genética Vegetal, en época del Virrey Cisneros. (Historia).

Minucias en que perdía tiempo el Cabildo de Córdoba.

Las primeras intervenciones quinirgicas con anestesia local.

Inspecciones de carne en época del Virrey Sobremonte.

Cabalgando en un silbido.

José Enrique Rodó.

Caña de Pescar.

* La Camiseta del Jefe de Policía. Córdoba: Editorial Tanke [s.a.], 159 pp.

48 En El hombre que se depiló la ingle, Viñole, por boca de Vagagá, formuló el siguiente vaticinio: "La odinofagia de mi pluma y de mi posición de lucha es tan real que cuando se haga la autopsia de mis verdades, dentro de cincuenta años, encontrarán inopinadamente razón a la razón de haber desparramado todas estas toxinas solubles. Al bosquejar la fisiología patológica de las intoxicaciones culturales se comprenderá que yo tuve valor de no ser confundido, como escritor y como erudito." (p. 99). 
* Jesús en una casa de departamentos. Córdoba: Editorial Tanke [s.a.] 190 pp.

Vidrio Molido. Viñoleanas.

El Vademécum del perfecto diputado.

Veronal o la vaca que tomaba cocaína.

La caligrafía de los juanetes en la arena de Mar del Plata.

El ojo que no tuvo paisajes.

$A$ usted le sale sangre.

* El Hombre que se depiló la ingle. Buenos Aires: Editorial Claridad [s.a.], $110 \mathrm{pp}$.

* Cien cabezas que se usan. Buenos Aires: Editorial Claridad [s.a.] $112 \mathrm{pp}$.

* Alambres de yeso. Buenos Aires: Editorial Claridad [s.a.] 95 pp.

* Mi disconformismo filosófico. Buenos Aires: Editorial Claridad [s.a.] 102 pp.

(se señalan con un asterisco los libros que han servido de base para la presente exposición.) 